Migraciones y movilidades de argentinos y argentinas: revisión crítica de un campo de estudios en desarrollo (1960-2020)

Patricia Jimena Rivero y Jose Navarro-Conticello

páginas / año 13 - n 31 Enero - Abril / ISSN 1851-992X/ 2021

http://revistapaginas.unr.edu.ar/index.php/RevPaginas

DOI: http://dx.doi.org/10.35305/rp.v13i31.474

\title{
Migraciones y movilidades de argentinos y argentinas: revisión crítica de un campo de estudios en desarrollo $(1960-2020)^{1}$
}

\author{
Migrations and mobilites of Argentines: critical review of a \\ developing field of studies (1960-2020)
}

\author{
Patricia Jimena Rivero \\ Instituto de Humanidades; \\ Consejo Nacional de Investigaciones Científicas y Técnicas; \\ Universidad Nacional de Córdoba (Agentina) \\ patriciaj.rivero@gmail.com
}

Jose Navarro-Conticello

Programa de Doctorado en Ciencias Humanas (Instituto de Estudios Humanísticos Juan Ignacio Molina y Facultad de Psicología);

Universidad de Talca (Chile).

jonavarro@utalca.cl

\begin{abstract}
Resumen
Se presenta una revisión crítica del desarrollo histórico del campo de estudios sobre migraciones y movilidades de argentinos y argentinas, entendido como el conjunto de publicaciones académicas destinadas a estudiar los procesos de emigración, inmigración y retorno de personas de esa nacionalidad. Con base en los factores predominantes que originan la movilidad, usualmente se distinguen tres etapas: fuga de cerebros (1960-1975), exilio político (1976-1983), migraciones económicas (1984-2004). En este estudio se propone una cuarta etapa, denominada "migraciones y movilidades" (2005-2020). El análisis permite establecer que el escenario global y los contextos políticos, sociales y económicos de Argentina han orientado la producción académica en determinadas direcciones, priorizándose ciertas categorías de análisis y desplazándose otras. Se destacan las contribuciones de los campos de estudios sobre ciencia, tecnología y sociedad y educación superior en el abordaje de las migraciones calificadas.
\end{abstract}

\footnotetext{
1 La primera autora agradece al Consejo Nacional de Investigaciones Científicas y Técnicas (CONICET) por financiar su investigación posdoctoral. El segundo autor agradece a la Comisión Nacional de Investigación Científica y Tecnológica (CONICYT) por financiar sus estudios de doctorado en Chile (CONICYT-PFCHA/Doctorado Nacional/2017-Folio N 21170178).
}

Esta obra está sujeta a la Licencia Reconocimiento-NoComercial-Compartirlgual 4.0 Internacional de Creative Commons. http://creativecommons.org/licenses/by-nc-sa/4.0/ 


\title{
Palabras Clave
}

campo de estudios; migraciones argentinas; movilidades; migración internacional; estudios migratorios; migración calificada.

\begin{abstract}
This paper presents a critical review of the historical development of the field of studies on Argentine migrations and mobilities, understood as the set of academic publications aimed at studying the processes of emigration, immigration and return of Argentine individuals. Based on the predominant factors that give rise to mobility, three stages are often distinguished: brain drain (1960-1975), political exile (1976-1983), economic migration (1984-2004). In this study it is proposed a fourth one, called "migrations and mobilities" (2005-2020). It is derived from the analysis that the global scenario, as well as the Argentine political, social and economic contexts, have oriented scholar production in certain directions, with certain categories of analysis being prioritized and others being displaced. The contributions of the fields of studies on science, technology and society and higher education in addressing skilled migrations are also highlighted.
\end{abstract}

\section{Keywords}

field of studies; migrations of Argentines; mobilities; international migration; migration studies; skilled migration.

\section{Introducción}

Este artículo propone una revisión del desarrollo histórico del campo de estudios sobre migraciones y movilidades de argentinos y argentinas, entendido como el conjunto de publicaciones académicas destinadas a explorar, describir y explicar los procesos de emigración, inmigración y retorno de personas de esa nacionalidad. No se pretende realizar un estudio exhaustivo de toda la literatura existente, sino abordar críticamente algunos de los contenidos y las ausencias más sobresalientes en el campo desde sus orígenes hasta la actualidad, en relación con los contextos predominantes en cada etapa, y proponer una actualización de las clasificaciones vigentes, que suelen organizar su desarrollo histórico en torno a tres etapas, incorporando una cuarta fase que se extendería hasta el presente.

Se parte aquí de un concepto amplio del fenómeno migratorio. La visión clásica de la migración suele consistir en el desplazamiento entre un lugar de origen y otro de destino que implica el reasentamiento permanente o relativamente duradero en este último. Pero existen procesos heterogéneos y variados en su direccionalidad, temporalidad, escala espacial y/o frecuencia. Estos han sido denominados con el término movilidades o mobilities (Sheller \& Urry, 2006, 2016; Faist, 2013; GlickSchiller \& Salazar, 2013). Este trabajo habla de migraciones y movilidades, comprendiendo ambas posibilidades. 


\section{Migraciones y movilidades de argentinos y argentinas: revisión crítica de un campo de estudios en desarrollo (1960-2020)}

Eguiguren (2017) define un campo de estudios como un conjunto dinámico de producción de conocimientos en el cual se define un objeto de estudio a través de preguntas sobre éste. Los campos de estudios visibilizan ciertos sujetos o aspectos, excluyendo otros (Carrillo \& Cortés, 2008) y los contextos de cada momento histórico inciden en esas priorizaciones y borramientos. Así, es importante estudiar las relaciones entre actores sociales, estados, academia y procesos migratorios para desnaturalizar y contextualizar categorías como migración o migrante (Eguiguren, 2017).

El campo de estudios sobre migraciones y movilidades de argentinos y argentinas tiene origen en las décadas de 1950 y 1960 y se inserta en el núcleo más amplio de los estudios migratorios en Argentina. Estos nacieron a finales del siglo XIX como expresión del interés de la intelectualidad por los alcances y efectos de la masiva inmigración europea (Domenech \& Pereira, 2017). Entre 1869 y 1914 la población extranjera en Argentina ascendió de 210 mil a 2,3 millones de personas, multiplicándose once veces (Pacecca \& Courtis, 2008; Benencia, 2012). En 1914 casi tres de cada diez residentes en el país eran de origen extranjero y apenas el $9 \%$ de esa población provenía de países limítrofes (Martínez \& Reboiras, 2008). De allí que en sus inicios la reflexión se haya concentrado en la inmigración, especialmente en la de ultramar.

Desde la segunda mitad del siglo XX el panorama migratorio de Argentina cambió. Por un lado, se diversificó la composición de la inmigración. Creció la cantidad de inmigrantes intrarregionales, que en 2019 ya representaban el 82,6\% de las personas extranjeras residentes en el país (OIM, 2020). También se intensificó la emigración, pasando el país de ser eminentemente receptor a desarrollar un doble perfil emisor-receptor de flujos migratorios (Benencia, 2012). El campo de estudios sobre migraciones y movilidades de argentinos y argentinas surgió del reconocimiento de la creciente complejidad del fenómeno y la necesidad de aportar teoría y evidencia al respecto.

Este trabajo parte de una pregunta: ¿cómo se han ido abordando históricamente las migraciones y movilidades de argentinos y argentinas, teniendo en cuenta los contextos económicos, sociales y políticos globales y nacionales? El objetivo es identificar algunos de los trabajos académicos cuyo tema principal son las migraciones y movilidades de personas argentinas, analizarlos, contextualizarlos en función de las clasificaciones vigentes que circunscriben su desarrollo histórico a tres etapas, y proponer una actualización de estas clasificaciones que se extienda hasta la actualidad.

\section{Método}

El artículo se basa en un diseño cualitativo que consiste en una revisión de literatura especializada, tipo de estudio que a partir del recorte y la sistematización de la producción previa en un campo de investigación provee información relevante para 


\section{Patricia Jimena Rivero y José Navarro-Conticello}

facilitar el desarrollo teórico y señalar direcciones futuras en la investigación (Webster \& Watson, 2002). Siguiendo la tipología de Grant \& Booth (2009), se trata de una revisión crítica o critical review, pues trasciende la mera descripción de las publicaciones identificadas e incluye un grado de análisis e innovación conceptual. Para la selección del corpus se hizo una búsqueda bibliográfica que de ninguna manera fue exhaustiva y a su vez estuvo probablemente permeada por las trayectorias académicas e investigativas de los autores del presente trabajo. Se aclara esto pues la pretensión del estudio no se corresponde con la exhaustividad ni el rigor propios de la metodología cuantitativa, sino que está vinculada con la búsqueda de la profundidad antes que el número, la riqueza conceptual y temática antes que la exhaustividad, y el reconocimiento de los propios sesgos interpretativos. Para la clasificación y el análisis de la bibliografía estudiada se utilizó un criterio de aplicación usual en el propio campo de estudios, basado en los factores predominantes como motores o causas de la emigración. Se complementó esta división, que distingue tres etapas en el desarrollo histórico del campo, con la propuesta de una hipotética cuarta etapa que se extendería hasta el presente, con el objeto de enriquecer y actualizar la discusión. En cada fase se identificaron categorías analíticas predominantes y otras menos desarrolladas. La producción del campo en cada etapa fue estudiada en relación con los contextos económicos, sociales y políticos relevantes en cada momento histórico en los planos nacional y global.

Si bien categorías como emigración, inmigración, retorno o repatriado conllevan sesgos propios del nacionalismo metodológico al restringir la discusión a los límites del Estado-nación (Martins, 1974; Wimmer \& Glick-Schiller, 2002), aquí se utilizan como tipos ideales, categorías analíticas que permiten vislumbrar los diferentes momentos del periplo migratorio. Tampoco la división en etapas y la diferenciación entre campos de estudios deben ser leídas como compartimentaciones rígidas, sino como clasificaciones operativas. En la realidad estas instancias admiten imbricaciones y zonas intermedias cuyos límites a menudo son borrosos.

\section{Resultados}

Con base en los factores predominantes que originan la emigración es posible distinguir tres grandes etapas en el desarrollo histórico del campo de estudios sobre migraciones y movilidades de argentinos y argentinas: la de la fuga de cerebros, de 1960 a 1975, la del exilio político, de 1976 a 1983, y la de las migraciones económicas, de 1984 a 2004. Cada una corresponde a un período histórico con ciertas condiciones contextuales. Esta clasificación tiene la ventaja de estar legitimada dentro del propio campo, pues distintos trabajos la han utilizado (Actis \& Esteban, 2008; Esteban, 2015; Rivero, 2019). Para enriquecer y actualizar la discusión, aquí se propondrá una hipotética cuarta etapa, desde 2005 hasta la 
Migraciones y movilidades de argentinos y argentinas: revisión crítica de un campo de estudios en desarrollo (1960-2020)

actualidad. A continuación, en la Tabla 1, se presenta un esquema del desarrollo histórico del campo según el análisis propuesto.

TABLA 1. Migraciones y movilidades de argentinos y argentinas: desarrollo histórico del campo de estudios (1960-2020)

\begin{tabular}{|c|c|c|c|c|}
\hline Período & $\begin{array}{l}\text { Nombre de } \\
\text { la etapa }\end{array}$ & Contexto nacional/global & $\begin{array}{l}\text { Categorías } \\
\text { analíticas } \\
\text { predominante } \\
\text { s }\end{array}$ & $\begin{array}{l}\text { Categorías } \\
\text { analíticas } \\
\text { olvidadas }\end{array}$ \\
\hline $\begin{array}{l}1960- \\
1975\end{array}$ & $\begin{array}{l}\text { Fuga de } \\
\text { cerebros }\end{array}$ & \multirow[t]{2}{*}{$\begin{array}{l}\text { Dictaduras } \\
\text { (Onganía, 1966, } \begin{array}{r}\text { militares } \\
\text { Videla, } \\
\text { 1976)/Políticas de captación } \\
\text { de talento en países } \\
\text { desarrollados }\end{array}\end{array}$} & \multirow[t]{2}{*}{$\begin{array}{l}\text { Exilios } \\
\text { (migración } \\
\text { calificada) }\end{array}$} & \multirow{2}{*}{$\begin{array}{l}\text { ¿Movimientos } \\
\text { transnacionales } \\
\text { con } \\
\text { Italia/España? } \\
\text { ¿Migraciones } \\
\text { Norte-Sur? } \\
\text { ¿Perfiles } \\
\text { migratorios } \\
\text { heterogéneos? }\end{array}$} \\
\hline $\begin{array}{l}1976- \\
1983\end{array}$ & Exilio político & & & \\
\hline $\begin{array}{l}1984- \\
2004\end{array}$ & $\begin{array}{l}\text { Migraciones } \\
\text { económicas }\end{array}$ & $\begin{array}{l}\text { Crisis económicas, políticas e } \\
\text { institucionales } \\
\text { (hiperinflación, } \\
\text { corralito)/Globalización }\end{array}$ & $\begin{array}{l}\text { Migraciones } \\
\text { económicas } \\
\text { (emigraciones) }\end{array}$ & \multirow{2}{*}{$\begin{array}{l}\text { Migraciones } \\
\text { calificadas } \\
\text { (estudiantes, } \\
\text { académicos, } \\
\text { científicos } \\
\text { investigadores) }\end{array}$} \\
\hline $\begin{array}{l}2005- \\
2020\end{array}$ & $\begin{array}{l}\text { Migraciones y } \\
\text { movilidades }\end{array}$ & $\begin{array}{l}\text { Ciclos de recuperación (2005- } \\
2015) \text { y crisis (2016-2019) de } \\
\text { la economía argentina, } \\
\text { fortalecimiento del sistema } \\
\text { de ciencia y tecnología, } \\
\text { programas de repatriación de } \\
\text { científicos/Globalización, } \\
\text { crisis global de } 2008\end{array}$ & $\begin{array}{l}\text { Migraciones } \\
\text { económicas } \\
\text { (emigración y } \\
\text { retorno) }\end{array}$ & \\
\hline
\end{tabular}

Fuente: Elaboración propia

\section{Conformación del campo de estudios: fuga de cerebros (1960-1975) y exilio político (1976-1983)}

Con más de seis décadas de discusión acerca de qué se entiende por migración calificada, encontramos quizás poco consenso en términos conceptuales, pero mayores acuerdos en cuanto a su aproximación empírica. Con el término recursos humanos altamente calificados se suele designar a aquellas "personas que poseen ciertas habilidades, conocimientos y capacidades, por lo que, contar con al menos nivel educativo terciario (licenciatura), o más, es la estrategia más común para delimitar a esta población" (Gandini, 2019: 378). En este trabajo referiremos a los recursos humanos altamente calificados como aquellas personas que han obtenido un grado mayor de calificación académica, expresado en el nivel de posgrado, y 


\section{Patricia Jimena Rivero y José Navarro-Conticello}

concretamente del grado de doctor. En América Latina, la emigración de este segmento de la población ha sido un tema de interés de la literatura especializada desde la década de 1960, principalmente en países del Cono Sur como Argentina, Chile y Uruguay, que se vieron especialmente afectados por el drenaje de esos recursos humanos (Didou, 2017). En esa época y en torno a esa temática empezó a conformarse el campo de estudios, cuya etapa inicial es la de la fuga de cerebros o brain drain.

Los debates sobre emigración calificada en América Latina pusieron a Argentina en el foco, por la cantidad de miembros de la élite intelectual de este país que debieron emigrar en dictaduras, un fenómeno denominado fuga de cerebros (Pellegrino, 2001). Tal categoría permitió identificar la pérdida de talento como una etapa emigratoria importante en la historia del país. Por primera vez se vincularon los debates sobre el desarrollo nacional y los procesos migratorios. Parte de la literatura de esta etapa dio cuenta de las consecuencias negativas del drenaje de estos perfiles en el desarrollo nacional (Albornoz, Fernández \& Alfaraz, 2002). Si bien la categoría tuvo posteriores revisiones con el surgimiento de nociones como intercambio de cerebros -brain exchange- o circulación de cerebros -brain circulation-, es útil para comprender las dinámicas centro-periferia y observar los fenómenos desde el Sur global (Vessuri, 2007; Castles \& Delgado, 2007; Delgado \& Márquez, 2007). Si de un lado había fuga, del otro había captación: países desarrollados como Estados Unidos y Canadá impulsaron políticas activas de incorporación de este tipo de migrantes.

Entre los primeros trabajos dedicados a esa línea están los de Horowitz (1962) y Houssay (1966) y una serie de estudios realizados por Oteiza (1965, 1967, 1969, 1971) sobre la emigración de población profesional, técnica y obrera especializada. En la misma época Palma (1974) publicó una investigación pionera sobre el retorno de personas altamente calificadas. A ellos se sumaron trabajos como los de Sito \& Stuhlman (1968), Slemenson (1970) y Oszlak \& Caputo (1973), este último específicamente sobre la emigración de profesionales médicos.

Una tendencia generalizada en esta primera etapa fue el foco en los condicionantes de la emigración calificada. Académicas y académicos contemporáneos que siguieron profundizando sobre las causas y consecuencias de la fuga de cerebros sostienen que esta etapa tiene puntos en común con la siguiente -del exilio político, pues ambas ocurrieron en contextos sociopolíticos represivos. Las dictaduras instauradas tras los golpes militares de Onganía en 1966 y Videla en 1976 coincidieron con un incremento sostenido de salidas hacia el exterior, y un rasgo que comparten ambos procesos emigratorios es la preponderancia de perfiles de alta calificación. En el primero, principalmente científicos y académicos; en el segundo, intelectuales, artistas, militantes políticos y sindicales (Jensen \& Lastra, 2014). Así, si bien la noción fuga de cerebros fue utilizada para explicar lo que ocurrió durante la primera etapa, también es plausible extenderlo a la segunda.

Mira \& Esteban (2008: 87) sostienen que el exilio político de 1976 fue la primera emigración argentina masiva, y por su composición representó una significativa 


\section{Migraciones y movilidades de argentinos y argentinas: revisión crítica de un campo de estudios en desarrollo (1960-2020)}

pérdida de capital humano. Además, subrayan que aquella fue la primera vez en la historia del país que la emigración superaba a la inmigración. No obstante, Graciarena (1986) advierte que no es fácil establecer una correlación directa entre autoritarismo y emigración calificada en Argentina. La medición del exilio político durante la represión se complejiza por la ausencia de datos fidedignos que permitan diferenciarlo de emigraciones calificadas por razones no políticas (García, 2008). Aun así, existe cierto consenso en torno a la relación entre autoritarismo y emigración calificada en países del Cono Sur durante la década de 1970 (Esteban, 2011).

La tendencia a considerar el exilio político como una variante de la migración calificada continuó durante la década de 1980, pero mientras algunas investigaciones insistieron en la necesidad de considerar la emigración como un fenómeno estructural y cuestionaron la especificidad de la emigración ocurrida durante las dictaduras de los años sesenta y setenta del siglo XX, otras incorporaron fuertemente en sus análisis la violencia política que caracterizó al país en ambos períodos (Jensen, 2005: 10).

Algunos trabajos pioneros de la etapa del exilio político que abordaron procesos emigratorios y de retorno son los de Maletta \& Szwarcberg (1985); Bertoncello \& Lattes (1986); Maletta, Szwarcberg \& Schneider (1986); Mármora \& Gurrieri (1988); Maletta (1988). Jensen (2005) también destaca las investigaciones de Eugenia Meyer, Eva Salgado y Pablo Yankelevich. Recientemente se han sumado estudios historiográficos (Jensen \& Coraza, 2009; Parisi, 2012; Lastra, 2013, 2014; Jensen \& Lastra, 2014).

Tras la recuperación de la democracia en Argentina, en 1983, varios trabajos abordaron cualitativamente los problemas de la adaptación laboral y social en los países de destino, las motivaciones de la decisión de retornar y los procesos de readaptación sociolaboral (Palomares, Castiglione \& Nejamkis, 2007: 25). El Estado argentino comenzó a implementar programas para repatriar personas exiliadas, con el fin de estimular el desarrollo científico y tecnológico. Debido a las deficiencias de estas políticas, algunos estudios analizaron el retorno desde la mirada del Estado. El retorno político fue, también, un tema de preocupación para organizaciones no estatales de defensa de los derechos humanos que asumieron un compromiso con el recibimiento y la asistencia a los retornados del exilio (Lastra, 2013).

Las migraciones calificadas fueron la categoría priorizada en las dos primeras etapas. Pero entonces ya existían otros tipos de movilidades. Actis \& Esteban (2007, 2008) y Esteban (2015) señalan que en 1950 había en España más de 6 mil argentinos y argentinas, y en 1970 cerca de 8 mil. Probablemente la mayoría de esos movimientos tuvieran un componente transnacional, fruto de las masivas migraciones españolas hacia Argentina de fines del siglo XIX y principios del XX, lo que hace "suponer la existencia de un conglomerado social diverso, en el que convivirían comerciantes y empresarios con artistas o deportistas, y desplazados políticos (peronistas y antiperonistas) junto a trabajadores vinculados a las redes familiares de la emigración hispana" (Actis \& Esteban, 2007: 221). Es posible que 
estos perfiles diversos hayan continuado desarrollándose durante estas dos primeras etapas; sin embargo, el campo no desarrolló categorías analíticas que dieran cuenta de ellos.

\section{Consolidación del campo de estudios: migraciones económicas (1984-2004)}

Tras el restablecimiento de la democracia surgió en Argentina un clima social de optimismo, aunque la situación económica era crítica: reducción del PIB, inflación sin precedentes y reducción salarial. Las razones principales de la emigración viraron desde la represión y persecución ideológica predominante en las etapas anteriores hacia la precarización provocada por las fuertes crisis económicoinstitucionales. Así, en la tercera etapa del desarrollo del campo se identifican dos períodos concretos, coincidentes con sendas crisis en el país de origen.

El primer período, de 1989 a 1992, coincidió con la crisis de la hiperinflación. Desde 1987 "el deterioro de la situación económica y la incapacidad estatal para preservar el valor de la moneda generaron un desastre hiperinflacionario que implicó la evaporación monetaria y la pérdida del control público sobre la economía" (Calvelo, 2008: 4). Las sedes consulares de España e Italia en las principales ciudades del país comenzaron a registrar largas filas para acceder a la ciudadanía ius sanguinis (Cacopardo, 1992). Otros destinos elegidos fueron Estados Unidos, Canadá y Australia (Pacecca \& Courtis, 2008).

El segundo período, de 1998 a 2001, estuvo marcado por el quiebre del sistema financiero, la devaluación de la moneda nacional, el deterioro de las condiciones de vida y una dramática crisis institucional que contribuyeron a acelerar la emigración (Pacecca \& Courtis, 2008). A diferencia de la crisis anterior, eminentemente económica, la del corralito -denominada así por la restricción bancaria de disposición de dinero en efectivo, plazos fijos y cuentas corrientes dispuesta por el gobierno de Fernando de la Rúa- significó también un desequilibrio políticoinstitucional cristalizado en el estallido social de diciembre de 2001.

Ambas crisis impactaron en el campo de estudios, que se renovó incorporando nuevas temáticas y preguntas de investigación, especialmente centradas en la emigración por razones económicas. La emigración de este período asumió un perfil más diverso que en las etapas anteriores, compuesto por distintos estratos de la clase media, provenientes del trabajo especializado y manual, el pequeño comercio, profesionales y estudiantes universitarios, entre otros sectores (Calvelo, 2008).

Novick \& Murias (2005); Novick (2007); Palomares, Castiglione \& Nejamkis (2007) analizaron la evolución de la emigración de comienzos del siglo XXI y constataron su composición heterogénea. Además, abordaron las políticas del Estado argentino frente a la emigración y el rol activo de otros actores sociales, como asociaciones de familiares de personas emigradas, funcionarios y funcionarias estatales. 


\section{Migraciones y movilidades de argentinos y argentinas: revisión crítica de un campo de estudios en desarrollo (1960-2020)}

La mayoría de los trabajos de la época indagaron en las motivaciones de la decisión de emigrar (González \& Merino, 2007; Schmidt, 2005, 2009). Algunas de estas causas están vinculadas a la realización personal y la búsqueda de oportunidades que el país ya no podía ofrecer. Según Jiménez (2011), ese anhelo de una vida mejor implicó una estrategia de reproducción social donde sectores de las clases medias argentinas emigraron ante la imposibilidad de mantener el estatus de clase en el país de origen, convirtiendo a la migración en el instrumento para evitar el desclasamiento.

Mira \& Esteban (2003), Actis \& Esteban (2007, 2008); Esteban (2007), Calvelo (2008; 2011) y Actis (2011), aportaron evidencia de un nuevo patrón migratorio o una complejización del existente: si bien el perfil de Argentina como receptor continuó vigente, se le sumó una arista de emisor de grandes flujos de migrantes, especialmente hacia Europa -sobre todo España-. Cacopardo, Maguid \& Martínez (2007) y Maguid \& Martínez (2008) profundizaron en esa línea y se preguntaron en qué medida este patrón fue compartido por el resto de los países sudamericanos.

España ha sido históricamente uno de los destinos predilectos de la emigración económica argentina. Esto explica que la mayoría de los estudios de esta etapa se hayan centrado en ese país. Sarrible (2000) y Vives-González (2011) analizaron la incorporación de estos migrantes en relación con las categorías administrativas que afectan a la inmigración y la ciudadanía en el lugar de destino. Otros abordaron la inserción sociolaboral, dando cuenta de los modos de incorporación al mercado de trabajo español e identificando nichos sectoriales de absorción y grado de consistencia entre ocupación y perfil educativo. Castellanos (2006) plantea que para comprender el éxito o fracaso de las trayectorias laborales en destino se debe atender a las especificidades territoriales de los mercados de trabajo, los proyectos migratorios y las situaciones de vulnerabilidad, entre ellas la propia condición de inmigrante. Estos factores, y las aspiraciones de progreso socioeconómico de la clase media, explicarían una gran cantidad de proyectos migratorios inconclusos o frustrados con movimientos de retorno. La idea de expectativas y anhelos no satisfechos también se encuentra en Palomares, Castiglione \& Nejamkis (2007) y con matices- en Gandini (2015).

Actis \& Esteban (2007, 2008) identificaron cinco etapas en las migraciones de Argentina a España: los pioneros, hasta 1974; la época del exilio, de 1975 a 1983; el inicio de la emigración económica, de 1984 a 1992; receso y reanudación de la emigración, de 1993 a 1999, y el éxodo del corralito, de 2000 a 2004. En un trabajo posterior (Esteban, 2015) el autor amplió el análisis hasta el año 2010 sumando la fase de "descenso de la emigración y... ¿retorno?", de 2005 a 2010 (p. 62). Rivero (2019) complementa ese estudio introduciendo una fase de descenso de la emigración, de 2005 a 2008, otra de boom del retorno, de 2009 a 2015, y una hipotética fase que incluiría re-emigraciones, circularidad y emigración de personas sin experiencia migratoria previa, de 2016 a 2017.

La mayoría de los trabajos de esta etapa analizaron las causas de la emigración argentina y, en algunos casos, los modos de incorporación sociolaboral en las 


\section{Patricia Jimena Rivero y José Navarro-Conticello}

sociedades de destino -inmigración-. El retorno, en cambio, permaneció como un área escasamente estudiada. La migración de retorno posterior a la crisis económica global iniciada en 2008 solo fue abordada en casos excepcionales. Un hecho que podría explicar esa vacancia es que, en los últimos años, las y los estudiosos de la migración en Argentina priorizaron dos elementos centrales: la inmigración extranjera, intensificada desde la recuperación de la economía argentina tras el shock de 2001, y la nueva política migratoria argentina, signada por la Ley de Migraciones 25.871, promulgada en 2004.

Un común denominador de los trabajos de esta etapa es que, aun reconociendo una mayor diversidad de perfiles que en períodos anteriores, la figura del migrante que se desplaza desde el Sur hacia el Norte y la idea del migrante económico permanecieron como elementos centrales, opacando otros tipos de movilidades. La crisis de 2001 fue el telón de fondo del mayor éxodo migratorio conocido en la historia argentina. Este incluyó variados perfiles y motivaciones, desde migraciones en sentido clásico hasta movilidades, migrantes económicos y desplazamientos de profesionales, científicos y recursos humanos altamente capacitados. Sin embargo el estudio sistemático de la migración calificada en Argentina fue escaso desde el retorno de la democracia, más allá de investigaciones como las de Lattes (1986), Leiva (1999) y Luchilo (2015), que analizaron el alcance de las distintas medidas estatales del Estado argentino destinadas a fomentar el retorno de investigadoras e investigadores residentes en el exterior.

Como indican Pedone \& Alfaro (2018), en algunos casos el perfil de los emigrados del 2001 combinaba estrategias laborales con las de perfeccionamiento educativo y, en otros, la búsqueda de ofertas educativas de posgrado que la región no ofrecía. La escasa inversión en ciencia y tecnología en la Argentina de la época afectó las posibilidades de desarrollar una carrera en esas áreas (Kreimer, 1998; Luchilo, 2015) e incidió en la emigración de perfiles de alta calificación.

La emigración calificada ha sido bastante constante a lo largo de la historia, inclusive en el marco de coyunturas y contextos favorables para el país. Como destaca Luchilo (2015), las condiciones y posibilidades en los países de destino tienen una influencia sobre las decisiones migratorias que es preciso reconocer (p. 182). Pellegrino (2001) plantea algo similar al señalar que la emigración calificada se ha convertido en una tendencia estructural en Argentina desde 1990, condición que, junto con las políticas de inmigración selectiva de los países centrales, caracteriza a los movimientos migratorios del período contemporáneo (p. 4).

Mientras en esta etapa el campo de estudios sobre migraciones y movilidades de argentinos y argentinas continuaba profundizando en las migraciones económicas, hubo otros que abordaron con fuerza la migración calificada. Se trata de los campos de estudios sobre ciencia, tecnología y sociedad (Vessuri, 1998; Kreimer, 1997, 1998, 2011; Spivak \& Hubert, 2012, 2014; Moreno, 2014, 2016) y educación superior (Luchilo, 2007a; García, 2008, 2009; Didou \& Gérard, 2009; Flores, 2009, 2010, 2011). Los primeros echaron luz sobre la dimensión social de las relaciones entre ciencia, tecnología e innovación, los mecanismos de circulación del 


\section{Migraciones y movilidades de argentinos y argentinas: revisión crítica de un campo de estudios en desarrollo (1960-2020)}

conocimiento y el impacto de los recorridos profesionales y de formación en el extranjero en la estructuración de las trayectorias científicas y los sistemas de ciencia y tecnología. Los segundos lograron algo similar, aunque tomando específicamente a la universidad como objeto de estudio, focalizando en las dinámicas de movilidad de estudiantes, docentes y personal no docente. Ambos analizaron la movilidad como un fenómeno característico del quehacer científico y universitario (Gaillard \& Gaillard, 1997).

\section{¿Una cuarta etapa? Migraciones y movilidades (2005-2020)}

Si bien no existe una categoría consensuada para denominar la producción del campo de estudios sobre migraciones y movilidades de argentinos y argentinas posterior a 2005, aquí se propone un esfuerzo en ese sentido. Esteban (2015) muestra que a partir de 2005 -su análisis se extiende hasta 2010- el volumen de la emigración argentina ha ido en descenso y se pregunta si se han producido procesos de retorno. Si bien el autor analiza las migraciones entre España y Argentina, lo cierto es que el impacto de la crisis global de 2008 planteó un punto de inflexión en la producción académica, volviendo el foco hacia el retorno.

La pregunta sobre si los emigrados de las crisis nacionales de 1989 y 2001 habían retornado al país de origen a raíz de la crisis global de 2008 aparece en algunos de los trabajos de esta etapa. No obstante, se trata de intentos más o menos aislados. En la búsqueda realizada a los fines de esta investigación solo fue posible identificar cuatro aportes en ese sentido, específicamente centrados en las migraciones y/o movilidades entre España y Argentina.

Uno de los primeros estudios en abordar los efectos de la crisis de 2008 fue el de Schmidt (2014), quien explora las motivaciones de retorno de argentinas y argentinos residentes en España. La autora plantea que no existe una razón determinante para emprender el retorno, sino múltiples factores que afectan a cada migrante en función de su proyecto migratorio, considerado en cada caso como único e intransferible.

Cerrutti \& Maguid $(2015,2016)$ estudian la migración argentina de retorno desde España en clave comparativa con otros grupos de migrantes latinoamericanos. Las autoras efectúan una cuantificación tentativa en volumen, analizando su perfil sociodemográfico y económico, y discutiendo su potencial contribución y reinserción en la sociedad argentina. Como se estima que el mayor número de retornados proviene de España, país que sufrió fuertemente los impactos de la crisis internacional, el estudio presta particular atención a ese proceso. Cerrutti, Maguid \& González (2018) presentaron recientemente un primer esbozo de datos primarios inéditos provenientes del proyecto Temporary vs. Permanent Migration (TEMPER) relevados en distintas provincias de Argentina sobre migrantes retornados. A partir de la aplicación de una encuesta, el estudio aporta evidencia sobre las trayectorias migratorias, laborales y familiares de personas retornadas, las motivaciones de la 


\section{Patricia Jimena Rivero y José Navarro-Conticello}

emigración y el retorno, la experiencia migratoria en España y el proceso de retorno desde su inicio hasta la actualidad.

Rivero (2019) y Cassain $(2016,2019)$ aplican la perspectiva transnacional, un enfoque que tiene casi tres décadas de desarrollo (Glick-Schiller, Basch \& SzantonBlanc, 1992; Wimmer \& Glick Schiller, 2002). Cassain estudia los diversos significados del retorno en las trayectorias migratorias de miembros de familias de argentinos y argentinas retornados de España a Argentina tras la crisis global de 2008. Rivero analiza las principales causas que intervienen en la migración de retorno de las y los argentinos que regresaron de España en el periodo 2008-2017, poniendo el foco de atención en los condicionantes individuales, relacionales y estructurales. La autora entiende al retorno como parte del ciclo migratorio y no como un simple acto de regreso al país de origen, y lo reconoce como un tipo complejo de movilidad imbricado con otros procesos de transformación social.

Navarro-Conticello \& Alonso-Meneses (2020) abordan la incorporación de migrantes argentinos y argentinas a un contexto de recepción inusual, marcado por la frontera entre México y Estados Unidos, y específicamente su adaptación a lo que los autores denominan un estilo de vida transfronterizo. Los autores proponen revisar categorías usuales en los estudios migratorios, como transnacional, a partir de las prácticas situadas de estas personas. Las y los migrantes estudiados realizan cruces cotidianos de esa frontera internacional en ambos sentidos y por diversas razones, constituyendo ejemplos de movilidad transnacional de corto radio espacial. En cuanto a los contextos globales, es importante tener en cuenta, tanto en esta etapa como en parte de la anterior, las implicancias de los fenómenos asociados a la globalización. Muchos de los desplazamientos que se producen en este marco responden a una tipología de migrantes en gran parte jóvenes, en búsqueda de nuevas experiencias de vida, cuyas trayectorias se asocian a una "cultura generacional y global de la movilidad" (Esteban, 2015: 138).

En esta etapa, como en la anterior, en el campo continuó predominando la categoría del migrante económico y la migración calificada no recibió gran atención, mientras que en los campos sobre ciencia, tecnología y sociedad y sobre educación superior esta última tuvo un importante desarrollo. Proveniente de estos, el estudio de Luchilo (2007a) propone tres etapas para dar cuenta de las causas que originaron la emigración de personal calificado en Argentina: fuga de cerebros, exilio y globalización. Al igual que la clasificación que se propone en el presente artículo, la división corresponde a los factores predominantes en cada caso como motores principales de la movilidad. Así, lo que el campo de estudios sobre migraciones y movilidades de argentinos y argentinas ha denominado la etapa de las migraciones económicas, estos otros campos lo han llamado la etapa de la globalización -desde mediados de la década de 1980 hasta la actualidad-, enfatizando en perfiles calificados: desplazamientos transitorios o temporales destinados al perfeccionamiento académico.

Un tema de reciente interés en el estudio de las migraciones calificadas en Argentina que ha sido estudiado por el campo de ciencia, tecnología y sociedad es el retorno, 


\section{Migraciones y movilidades de argentinos y argentinas: revisión crítica de un campo de estudios en desarrollo (1960-2020)}

específicamente los procesos de reinserción de científicos, científicas, académicas y académicos que residían en el exterior (Luchilo, 2007, 2007a; Jonkers \& CruzCastro, 2013; Moreno, 2014, 2016). Especialmente en el marco de la actual política de repatriación y vinculación de científicos de Argentina, el Programa RAÍCES (Red de Argentinos Investigadores y Científicos en el Exterior) contiene contribuciones como las de Luchilo (2011, 2015); Spivak \& Hubert (2014); MINCYT (2015); Bayle (2014, 2015); Serra (2018) y Rivero \& Trejo (2019).

No obstante, son pocos los estudios que tienen como unidad de análisis a los propios repatriados. Esfuerzos en esa dirección son los trabajos de Spivak L'Hoste \& Hubert (2014), Rivero (2018), Serra (2018)y Rivero \& Trejo(2019). Tanto Serra como Spivak \& Hubert abordan las prácticas y subjetividades en el marco de experiencias particulares de retorno a través de un análisis de trayectorias académicas y de vida. Rivero (2018) analiza los efectos del Programa RAÍCES en el desarrollo del sistema científico y tecnológico argentino, observando los procesos de internacionalización a través de las trayectorias científicas de investigadores retornados. Rivero \& Trejo (2019) estudian cómo inciden los programas de repatriación de científicos de México y Argentina en los procesos de reinserción en universidades y organismos de ciencia y tecnología, analizando especialmente las trayectorias científicas de jóvenes en etapa temprana de la carrera académica o early career researchers, los perfiles mayoritarios que suelen repatriar dichos programas.

\section{Conclusiones}

El artículo propuso una revisión crítica del desarrollo histórico del campo de estudios sobre migraciones y movilidades de argentinos y argentinas desde sus orígenes hasta el presente. Para ello se empleó una clasificación usual en la literatura especializada, la cual divide la producción en tres etapas de acuerdo con el motivo principal de la emigración, desde el surgimiento del campo en la década de 1960 hasta el año 2004. Recordando que esta división no es taxativa, sino simplemente operativa, aquí se propuso una cuarta etapa que se extendería desde 2005 hasta el presente. Con ello se buscó enriquecer y actualizar la discusión, aportando un grado de innovación sin alterar la estructura de un esquema que goza de consenso en la comunidad especializada.

Una de las conclusiones que derivan de los resultados es que el campo de estudios, a seis décadas de su conformación, aún se encuentra en desarrollo. Esto no significa que no se hayan registrado avances sustantivos. Al contrario, el repaso crítico de la producción en cada una de las etapas permite comprender que existen varias líneas de investigación y categorías analíticas que han sido ampliamente trabajadas a lo largo del tiempo, lo cual denota un grado de avance del conocimiento. No obstante, hay otras que han sido olvidadas o poco estudiadas, vacancias que es necesario llenar a través de nuevas preguntas de investigación. 
El análisis por etapas ha permitido reconstruir el rumbo de la producción académica en su desarrollo histórico. En tal sentido, los condicionantes macro-estructurales han orientado la producción del campo de estudios. Los contextos políticos, económicos y sociales que ha atravesado Argentina a lo largo de estas seis décadas, así como otros procesos de mayor alcance a escala global, han tenido incidencia en el campo, que en cada etapa ha priorizado ciertas categorías de análisis, desplazando $\mathrm{u}$ omitiendo otras.

En las dos primeras etapas -fuga de cerebros, de 1960 a 1975, y exilio político, de 1976 a 1983- se situó el foco en los perfiles migratorios de alta calificación, dejando poco espacio al análisis de otro tipo de migrantes y categorías analíticas. Por ejemplo, como indican Actis \& Esteban $(2007,2008)$ y Esteban $(2015)$, en esos períodos vivían en España argentinos y argentinas de perfiles variados, que en muchos casos habían arribado a Europa en el marco de movimientos transnacionales iniciados con las migraciones previas de sus ancestros españoles a Argentina. Pero no hay estudios al respecto. El marcado interés por la pérdida de talentos por sobre otras categorías de análisis posiblemente esté relacionado con el impacto que en la Argentina de la época comenzaba a tener el éxodo de algunos de sus recursos humanos más altamente calificados, en términos de desarrollo nacional.

La etapa de las migraciones económicas -de 1984 a 2004- se ha perfilado como una fase de consolidación del campo, pues en ella se complejizó la discusión precedente y se generaron nuevas categorías y preguntas de investigación. Buena parte de los movimientos migratorios producidos en el marco del éxodo posterior a la crisis argentina de 2001 corresponden a migraciones económicas, aunque también hubo emigraciones por razones de estudio y perfeccionamiento académico (Pedone \& Alfaro, 2018). Si bien el grueso del campo solo ha puesto el foco en las migraciones económicas, algunos trabajos empíricos han demostrado que en ese flujo, tanto al momento de emigrar como de retornar, han coexistido ambos tipos de desplazamientos (Rivero, 2019).

Una cuestión no menor respecto de la discusión sobre los flujos migratorios iniciados en las últimas crisis económicas es que todavía requieren ser profundizadas, pues refieren a procesos aún en desarrollo. Algunas personas emigradas siguen residiendo en el exterior, otras han retornado y otras continúan realizando desplazamientos continuos de ida y vuelta. Los análisis sobre este último tipo de dinámicas, las migraciones circulares, casi no han sido trabajadas dentro del campo de estudios. Sin embargo, hay evidencia empírica de su existencia (Rivero, 2018a, 2019). Es imposible comprender a fondo las consecuencias de los procesos emigratorios producidos en las crisis más importantes que ha sufrido la Argentina en su historia reciente -la de 1989 y la de 2001- sin nuevas preguntas, categorías y perspectivas de investigación que incorporen a cada vez más actores y actoras.

Uno de los aportes de este estudio es que propone la existencia de una cuarta etapa en el desarrollo del campo, la cual se extendería aproximadamente desde 2005 hasta la actualidad, denominada migraciones y movilidades. Esta incluiría trabajos que 


\section{Migraciones y movilidades de argentinos y argentinas: revisión crítica de un campo de estudios en desarrollo (1960-2020)}

analizan movimientos variados en cuanto a su frecuencia, temporalidad o escala espacial. No obstante, en esta hipotética etapa, como en la anterior, continuó predominando la categoría del migrante económico y la migración calificada -que en las primeras dos etapas había sido preponderante- ya no recibió gran atención. Uno de los fenómenos destacados en la cuarta etapa propuesta es la migración de retorno. Si bien los estudios son escasos, han aportado nuevas preguntas de investigación, perspectivas teórico-metodológicas y referentes empíricos. Así, es posible afirmar que el inicio del siglo XXI plantea una nueva dirección en el campo de estudios, más dinámica. Pareciera imponerse el imperativo de incorporar nuevas herramientas que permitan comprender las migraciones y movilidades de argentinos y argentinas como procesos complejos. El uso cada vez más extendido de la perspectiva transnacional y los análisis multiescalares en los estudios migratorios internacionales (Feldman-Bianco, 2015; Çağlar \& Glick-Schiller, 2018) parece haber impactado en el campo de estudios sobre migraciones y movilidades de argentinos $\mathrm{y}$ argentinas, permitiendo vincular escalas locales y globales y cuestionar las dicotomías entre origen/destino, emigración/retorno, expatriación/repatriación (Cavalcanti \& Parella, 2013).

En relación con el perfil calificado, el artículo hace una contribución inusual a la discusión sobre el desarrollo del campo, incorporando investigaciones provenientes de dos campos no especializados en migraciones: los estudios sobre ciencia, tecnología y sociedad y sobre educación superior. Estos han aportado evidencia de los distintos tipos de desplazamientos de personas con altos niveles de calificación y las motivaciones que los originan. El incremento de su movilidad y la corta temporalidad de sus estadías sugieren la necesidad de incorporar nuevas categorías analíticas que den cuenta de esos fenómenos (Mármora, 1998).

En cuanto a los factores contextuales, a nivel nacional se observan vaivenes constantes entre situaciones de recuperación económica, como en el período 20052015, y de crisis, como la de 2016 al presente. A eso se suman acontecimientos de escala global, desde los efectos de la globalización a crisis financieras como la de 2008 y recientemente la pandemia de COVID-19. Es de esperar que estos fenómenos continúen complejizando y diversificando los movimientos migratorios, lo cual renueva el desafío de actualizar las herramientas para comprenderlos y -como sugieren los aportes de los campos sobre ciencia, tecnología y sociedad y educación superior- la necesidad de superar la compartimentación de los estudios y avanzar hacia la transdisciplinariedad.

\section{Bibliografía}

Actis, Walter (2011). Migraciones Argentina-España. Características de los distintos "ciclos" migratorios, sus inserciones en España y el impacto de la crisis actual. En Cynthia Pizzarro (coord.), Migraciones internacionales contemporáneas. Estudios para el debate. Buenos Aires: CICCUS. 
Actis, Walter \& Esteban, Fernando (2007). Argentinos en España ("sudacas" en tierras "gallegas"): el estado de la cuestión. En Susana Novick (dir.), Sur-Norte. Estudios sobre la reciente emigración de argentinos. Buenos Aires: Catálogos.

Actis, Walter \& Esteban, Fernando (2008). Argentinos en España: inmigrantes, a pesar de todo. Migraciones, 23, 79-115.

Albornoz, Mario, Fernández, Ernesto \& Alfaraz, Claudio (2002). Hacia una nueva estimación de la "fuga de cerebros". Redes, 9(18), 63-84.

Bayle, Paola (2014). El Programa RAÍCES (Red de Argentinos Investigadores y Científicos en el Exterior) frente a la transferencia y circulación de conocimientos en la periferia: ¿Un nuevo patrón de cooperación científica internacional? Ponencia presentada en Conferencia FLACSO-ISA: Poderes globales y regionales en un mundo en cambio. Buenos Aires, 23-35 de Julio.

Bayle, Paola (2015). Mapping the Return of Argentine Researchers. Science, Technology and $\quad$ Society, 20(3), 435-449. https://doi.org/10.1177/0971721815597165

Benencia, Roberto (2012). Perfil migratorio de Argentina 2012. Buenos Aires: OIM.

Bertoncello, Rodolfo \& Lattes, Alfredo (1986). Medición de la emigración de argentinos a partir de la información nacional. En Alfredo Lattes \& Enrique Oteiza (comps.), Dinámica migratoria argentina (1955-1984): democratización y retorno de expatriados. Buenos Aires: CEAL.

Cacopardo, María (1992). La emigración potencial de jóvenes italoargentinos. Estudios Migratorios Latinoamericanos, 7(22), 453-495.

Cacopardo, María, Maguid, Alicia \& Martínez, Rosana (2007). La nueva emigración de latinoamericanos a España: el caso de los argentinos desde una perspectiva comparada. Papeles de Población, 13(51), 9-44.

Çağlar, Ayșe \& Glick-Shiller, Nina (2018). Migrants \& City-Making. Dispossession, Displacement, and Urban Regeneration. Durham y Londres: Duke University Press.

Calvelo, Laura (2008). La emigración argentina y su tratamiento público (19602003). Ponencia presentada en III Congreso de la ALAP. Córdoba, 24-26 de Septiembre.

Calvelo, Laura (2011). Crisis y emigración: la emigración de los argentinos entre 1960 y 2002. Buenos Aires: Ministerio del Interior.

Carrillo, María \& Cortés, Almudena (2008). Por la migración se llega a Ecuador: una revisión de los estudios sobre la migración ecuatoriana en España. En Gioconda 


\section{Migraciones y movilidades de argentinos y argentinas: revisión crítica de un campo de estudios en desarrollo (1960-2020)}

Herrera \& Jacques Ramírez (eds.), América Latina migrante: Estado, familias, identidades. Quito: FLACSO/Ministerio de Cultura.

Cassain, Laura (2016). Migration trajectories and return processes: An exploration of multi-generational family experiences between Spain and Argentina.

Transnational Social Review, 6(1-2), 41-59.

https://doi.org/10.1080/21931674.2016.1180843

Cassain, Laura (2019). Volver. Trayectorias migratorias y procesos de retorno de España a Argentina [tesis]. Madrid: UCM.

Castellanos, Mari (2006). "Si te parás a pensar, perdimos". Relatos de vida y expectativas frustradas de la inmigración argentina en España. Estudios Migratorios Latinoamericanos, 60, 363-412.

Castles, Stephen \& Delgado, Raúl (2007). Migración y desarrollo: perspectivas desde el sur. Zacatecas: UAZ.

Cavalcanti, Leonardo \& Parella, Sonia (2013). El retorno desde una perspectiva transnacional. Revista Interdisciplinar da Mobilidade Humana, 21(41), 9-20. http://dx.doi.org/10.1590/S1980-85852013000200002

Cerrutti, Marcela \& Maguid, Alicia (2015). El retorno reciente de argentinos desde el exterior: ¿Un tema para la agenda migratoria? Ponencia presentada en XII Jornadas Argentinas de Estudios de Población. Salta, 16-18 de Septiembre.

Cerrutti, Marcela \& Maguid, Alicia (2016): Crisis económica en España y el retorno de inmigrantes sudamericanos. Migraciones Internacionales, 8(3), 155-189.

Cerrutti, Marcela, Maguid, Alicia \& González, Amparo (2018). Lejana tierra mía: dimensiones del retorno argentino desde España. Ponencia presentada en Congreso Internacional de ALAP. Puebla, 23-26 de octubre.

Delgado, Raúl \& Márquez, Humberto (2007). Repensar la relación entre migración y desarrollo a partir del caso de México. Liminar, 5(2), 8-25. http://dx.doi.org/10.29043/liminar.v5i2.248

Didou, Sylvie \& Gérard, Etienne (eds.) (2009). Fuga de cerebros, movilidad académica y redes científicas. Perspectivas latinoamericanas. México: IESALC/CINVESTAV/IRD.

Didou, Sylvie (2017). La internacionalización de la educación superior en América Latina: transitar de lo exógeno a lo endógeno. México: Centro Cultural Tlatelolco.

Domenech, Eduardo \& Pereira, Andrés (2017). Estudios migratorios e investigación académica sobre las políticas de migraciones internacionales en Argentina. Íconos, 58, 83-108. http://dx.doi.org/10.17141/iconos.58.2017.2487 
Eguiguren, María (2017). Los estudios de la migración en Ecuador: del desarrollo nacional a las movilidades. Íconos, 58, 59-81. http://dx.doi.org/10.17141/iconos.58.2017.2497

Esteban, Fernando (2007). Inmigración argentina a España (2000-2005): claves interpretativas del proceso migratorio y la integración socioeconómica. Estudios Migratorios Latinoamericanos, 21, 367-415.

Esteban, Fernando (2011). La movilidad de profesionales y estudiantes universitarios latinoamericanos y caribeños a países de la OCDE. Papeles de población, 17(69), 105-149.

Esteban, Fernando (2015). El sueño de los perdedores: cuatro décadas de migraciones de argentinos a España (1970-2010). Buenos Aires: Teseo.

Esteban, Fernando (2018). Argentinos en España: una comparación entre la inserción laboral de exiliados y migrantes económicos. Avances del Cesor, 15(18), 45-78.

Faist, Thomas (2013). The Mobility Turn: A New Paradigm for the Social Sciences? Ethnic and Racial Studies, 36(11), 1637-1646. https://doi.org/10.1080/01419870.2013.812229

Feldman-Bianco, Bela (2015). Desarrollos de la perspectiva transnacional: migración, ciudad y economía política. Alteridades, 25(50), 13-26.

Flores, Patricia (2009). Análisis de la dinámica de movilidad internacional de graduados universitarios argentinos [tesis]. Buenos Aires: UNGS.

Flores, Patricia (2010). Principales evidencias de la movilidad internacional de graduados universitarios argentinos. Revista Iberoamericana sobre Ciencia, Tecnología y Sociedad, 14(5), 63-85.

Flores, Patricia (2011). Los flujos de movilidad internacional de estudiantes y graduados universitarios argentinos en el contexto de internacionalización de la educación superior. En Lucas Luchilo (coord.), Más allá de la fuga de cerebros. Movilidad, migración y diásporas de argentinos calificados. Buenos Aires: Eudeba.

Gaillard, Jacques \& Gaillard, Anne Marie (1997). The international mobility of brains: exodus or circulation? Science, Technology \& Society, 2(2), 195-228. https://doi.org/10.1177/097172189700200202

Gandini, Luciana (2015). ¿Escapando de la crisis? Un estudio comparativo de trayectorias laborales de migrantes argentinos en la Ciudad de México y Madrid. Cuernavaca: UNAM. 


\section{Migraciones y movilidades de argentinos y argentinas: revisión crítica de un campo de estudios en desarrollo (1960-2020)}

Gandini, Luciana (2019). Explicaciones de la migración calificada: el papel de las mujeres desde la experiencia norteamericana. Estereotipos, sesgos y desafíos. Norteamérica, $14(1)$ 369-390. http://dx.doi.org/10.22201/cisan.24487228e.2019.1.371

García, Ana (2008). Políticas públicas frente a la "fuga de cerebros". Reflexiones a partir del caso argentino. Revista de la Educación Superior, 37(148), 111-121.

García, Ana (2009). La movilidad académica y estudiantil: reflexiones sobre el caso argentino. En Sylvie Didou \& Etienne Gérard (eds.), Fuga de cerebros, movilidad académica, redes científicas. Perspectivas latinoamericanas. México: Cinvestav.

Glick-Schiller, Nina, Basch, Linda \& Szanton-Blanc, Cristina (1992). Towards a Definition of Transnationalism: Introductory Remarks and Research Questions. En Nina Glick-Schiller (ed.), Towards a Transnational Perspective on Migration: Race, Class, Ethnicity and Nationalism Reconsidered. Nueva York: NYAS.

Glick-Schiller, Nina \& Salazar, Noel (2013). Regimes of Mobility Across the Globe. Journal of Ethnic and Migration Studies, 39(2), 1-18. https://doi.org/10.1080/1369183X.2013.723253

Grant, Maria \& Booth, Andrew (2009). A typology of reviews: an analysis of 14 review types and associated methodologies. Health Information and Libraries Journal, 26, 91-108. http://dx.doi.org/10.1111/j.1471-1842.2009.00848.x

González, Elda \& Merino, Asunción (2007). Historias de acá. Trayectoria migratoria de los argentinos en España. Madrid: CSIC.

Graciarena, Jorge (1986). Prólogo. En A. Lattes \& E. Oteiza (comps.), Dinámica migratoria argentina (1955-1984): democratización y retorno de expatriados. Ginebra: UNRISD/CENEP.

Horowitz, Morris (1962). La emigración de técnicos y profesionales argentinos. Buenos Aires: Editorial del Instituto.

Houssay, Bernardo (1966). La emigración de científicos, profesionales y técnicos de la Argentina. Ciencia Interamericana, 7, 4-12.

Jensen, Silvina (2005). La historiografía del último exilio argentino: un territorio en construcción. Ponencia presentada en X Jornadas Interescuelas/Departamentos de Historia. Rosario, 22-23 de Septiembre.

Jensen, Silvina \& Coraza, Enrique (2009). Los pioneros de la inmigración latinoamericana a España: exiliados uruguayos y argentinos. En Ángeles Escrivá, Anastasia Bermúdez \& Natalia Moraes (eds.), Migración y Participación Política. Madrid: CSIC. 


\section{Patricia Jimena Rivero y José Navarro-Conticello}

Jensen, Silvina \& Lastra, Soledad (eds.) (2014). Exilios: Militancia y represión. Nuevas fuentes y nuevos abordajes de los destierros de la Argentina de los años setenta. La Plata: EDULP.

Jiménez, Cecilia (2011). Desclasamiento y reconversiones en las trayectorias de los migrantes argentinos de clases medias [tesis]. Madrid: UCM.

Jonkers, Koen \& Cruz-Castro, Laura (2013). Research upon return: The effect of international mobility on scientific ties, production and impact. Research Policy, 42(8), 1366-1377. https://doi.org/10.1016/j.respol.2013.05.005

Kreimer, Pablo (1997). Migration of Scientists and the Building of a Laboratory in Argentina. Science, Technology and Society, 2(2), 229-259. https://doi.org/10.1177/097172189700200203

Kreimer, Pablo (1998). Migración de científicos y estrategias de reinserción, en Jorge Charum \& Jean-Baptiste Meyer (eds.), El nuevo nomadismo científico. La perspectiva latinoamericana. Bogotá: ESAP.

Kreimer, Pablo (2011). Internacionalização e tensões da ciência latino-americana. Ciência e Cultura, 63(2), 56-59. http://dx.doi.org/10.21800/S0009$\underline{67252011000200018}$

Lastra, María (2013). ¿Volver al hogar? La experiencia del retorno de los exiliados argentinos. Andamios, 10, 321-344. https://doi.org/10.29092/uacm.v10i21.363

Lastra, María (2014). Los retornos del exilio en Argentina y Uruguay: Una historia comparada de las políticas y tensiones en la recepción y asistencia en las posdictaduras (1983-1989) [tesis]. La Plata: UNLP.

Lattes, Alfredo (1986). Dinámica migratoria argentina (1955-1984): democratización y retorno de expatriados. Ginebra: UNRISD/CENEP.

Leiva, María (1999). Políticas de recuperación y vinculación de argentinos en el exterior: valorización del patrimonio nacional e integración regional [tesis]. Buenos Aires: UBA.

Luchilo, Lucas (2007). Migración de retorno: el caso argentino, Working Paper No. 39. Buenos Aires: CENEP.

Luchilo, Lucas (2007a). Migraciones de científicos e ingenieros latinoamericanos: fuga de cerebros, exilio y globalización. En Jesús Sebastián (ed.), Claves del desarrollo científico y tecnológico de América Latina. Madrid: Siglo XXI/Fundación Carolina.

Luchilo, Lucas (ed.) (2011). Más allá de la fuga de cerebros: movilidad, migración y diásporas de argentinos calificados. Buenos Aires: Eudeba. 


\section{Migraciones y movilidades de argentinos y argentinas: revisión crítica de un campo de estudios en desarrollo (1960-2020)}

Luchilo, Lucas (2015). Políticas argentinas de retorno y de vinculación: rupturas y continuidades. En Jean-Baptiste Meyer, Diáspora: hacia la nueva frontera. Montevideo: Universidad de la República.

Maguid, Alicia \& Martínez, Rosana (2008). La emigración reciente de sudamericanos a Estados Unidos ya España: El caso de los argentinos. Ponencia presentada en III Congreso de la ALAP. Córdoba, 24-26 de Septiembre.

Maletta, Héctor (1988). Del pasivo al activo: una política para los emigrados de América Latina. Estudios Migratorios, 3(10), 497-521.

Maletta, Héctor \& Szwarcberg, Frida (1985). Migración de retorno a la Argentina: problemas económicos y psicosociales. Informe de investigación al proyecto hemisférico de migración. Buenos Aires: Dirección Nacional de Migraciones.

Maletta, Héctor, Szwarcberg, Frida \& Schneider, Rosalía (1986). Exclusión y reencuentro: aspectos psicosociales del retorno de los exiliados a la Argentina. Revista Estudios Migratorios, 1(3), 293-319.

Mármora, Lelio (1998). Políticas y programas de migraciones de recursos humanos calificados. En Jorge Charum \& Jean-Baptiste Meyer (eds.), El nuevo nomadismo científico. La perspectiva latinoamericana. Bogotá: ESAP.

Mármora, Lelio \& Gurrieri, Jorge (1988). Retorno en el Río de la Plata (las respuestas sociales frente al retorno en Argentina y Uruguay). Estudios Migratorios Latinoamericanos, 3(10), 467-495.

Martínez, Jorge \& Reboiras, Leandro (2008). Impacto social y económico de la inserción de los migrantes en tres países seleccionados de Iberoamérica. Serie Población y Desarrollo 83. Santiago de Chile: CELADE.

Martins, Herminio (1974). Time and theory in Sociology. En John Rex (ed.), Approaches to Sociology: An introduction to major trends in british sociology. Londres/Boston: Routledge/Kegan Paul.

MINCYT (Ministerio de Ciencia, Tecnología e Innovación Productiva) (2015). Programa Raíces, una política de estado. Buenos Aires: MINCYT.

Mira, Guillermo \& Esteban, Fernando (2003). El flujo que no cesa: aproximación a las razones, cronología y perfil de los argentinos radicados en España (1975-2001). Historia Actual Online, 2(1), 33-44.

Mira, Guillermo \& Esteban, Fernando (2008). Migraciones y exilios: memorias de la historia argentina reciente a través del cine. Athenea Digital, 14, 83-104. https://doi.org/10.5565/rev/athenead/v0n14.490 
Moreno, Verónica (2014). Reflexiones sobre la migración de científicos argentinos hacia los Estados Unidos. ¿Es pertinente el modelo centro-periferia para analizarla? Odisea. Revista de Estudios Migratorios, 1, 144-169.

Moreno, Verónica (2016). Sobre las diferencias salariales y la atomización de los agentes en la migración calificada. Ciencia, Docencia Tecnología, 27(53), 1-32.

Navarro-Conticello, Jose \& Alonso-Meneses, Guillermo (2020). Inmigrantes argentinos en San Diego y Tijuana. Estrategias de adaptación a un estilo de vida transfronterizo. Migraciones, 49 59-83. https://doi.org/10.14422/mig.i49.y2020.003

Novick, Susana (ed.) (2007). Sur-Norte. Estudios sobre la emigración reciente de argentinos. Buenos Aires: Catálogos.

Novick, Susana \& Murias, Gabriela (2005). Dos estudios sobre la emigración reciente en la Argentina. Documentos de Trabajo No 42. IIGG. Buenos Aires: UBA.

OIM (Organización Internacional para las Migraciones) (2020). Tendencias Migratorias en América del Sur. Informe Migratorio Sudamericano N³ Año 2020.

Oszlak, Oscar \& Caputo, Dante (1973). La emigración del personal médico de América Latina a Estados Unidos. Una interpretación alternativa. Buenos Aires: OMS.

Oteiza, Enrique (1965). La emigración de ingenieros en la Argentina. Un caso de "brain drain" latinoamericano. Revista Internacional del Trabajo, 72(16). https://doi.org/10.2307/3466065

Oteiza, Enrique (1967). La emigración de personal altamente calificado en la Argentina. Un caso de "brain drain" latinoamericano. Buenos Aires: Editorial del Instituto.

Oteiza, Enrique (1969). Emigración de profesionales, técnicos y obreros calificados argentinos a los Estados Unidos. Análisis de sus fluctuaciones (actualizado con los datos del período julio 1966 a junio 1968). Buenos Aires: Editorial del Instituto.

Oteiza, Enrique (1971). Emigración de profesionales, técnicos y obreros calificados argentinos a los Estados Unidos: análisis de la fluctuación de la emigración bruta, julio 1950 a junio 1970. Desarrollo Económico, 10(39/40), 429-454.

Pacecca, María \& Courtis, Corina (2008). Inmigración contemporánea en Argentina: dinámicas y políticas. Santiago de Chile: ONU/CEPAL/CELADE.

Palma, Sara (1974). Emigración y retorno de profesionales: el impacto de los estudios en el exterior. San Carlos de Bariloche, Argentina: Fundación Bariloche. 


\section{Migraciones y movilidades de argentinos y argentinas: revisión crítica de un campo de estudios en desarrollo (1960-2020)}

Palomares, Marta, Castiglione, Celeste \& Nejamkis, Lucila (2007). Emigración reciente de argentinos: el regreso a casa. En Susana Novick (dir.), Sur-Norte. Estudios sobre la reciente emigración de argentinos. Buenos Aires: Catálogos.

Parisi, María (2012). El desexilio de los ArgenMex: un estudio sobre las representaciones sobre el exilio y el retorno al país en los hijos de exiliados políticos. Ponencia presentada en I Jornadas de Trabajo sobre Exilios Políticos del Cono Sur en el siglo XX. La Plata, 26-28 de Septiembre.

Pedone, Claudia \& Alfaro, Yolanda (2018). La migración cualificada en América Latina: una revisión de los abordajes teóricos metodológicos y sus desafíos. Periplos, 2(1), 3-18.

Pellegrino, Adela (2001). Éxodo, movilidad y circulación: nuevas modalidades de la migración calificada. Notas de Población, 73, 129-162.

Rivero, Patricia (2018). La internacionalización de la ciencia y la tecnología en Argentina: evaluación del Programa RAÍCES a partir de las trayectorias científicas de los investigadores repatriados (2008-2020). [Documento inédito].

Rivero, Patricia (2018a). El retorno en el régimen de movilidad global: un análisis a partir de las trayectorias de movilidad de los argentinos que retornan de España. Cuadernos Latinoamericanos, 30(54), 6-21.

Rivero, Patricia (2019). Migración de retorno desde una perspectiva transnacional. Un análisis multinivel sobre los procesos de decisión de los argentinos que retornan desde España [tesis]. Córdoba: UNC.

Rivero, Patricia \& Trejo, Alma (2019). Procesos de reinserción de investigadores en etapa temprana de la carrera académica: los programas de repatriación en México y Argentina. Ponencia presentada en III Congreso Nacional de Sociología Pre-Alas. San Juan, 4-6 de Septiembre.

Sarrible, Graciela (2000). El regreso a Europa: argentinos en España. Scripta Nova. Revista Electrónica de Geografía y Ciencias Sociales, 59.

Serra, María (2018). Trayectorias académicas y migraciones altamente calificadas: una aproximación al caso de los científicos y científicas retornados a la ciudad de Santa Fe (2001-2015). Estudios Sociales, 55(2), 27-55. https://doi.org/10.14409/es.v55i1.6723

Sheller, Mimi \& Urry, John (2006). The new mobilities paradigm. Environment and Planning A, 38, 207-226. https://doi.org/10.1068/a37268

Sheller, Mimi \& Urry, John (2016). Mobilizing the new mobilities paradigm. Applied Mobilities, 1(1), 10-25. https://doi.org/10.1080/23800127.2016.1151216 
Schmidt, Hebe (2014). Crónicas del retorno. Motivaciones y estrategias del colectivo argentino en España. Madrid: Comunidad de Madrid.

Schmidt, Susana (2005). Causas de la emigración argentina actual a España en el discurso de los argentinos radicados en Madrid. En Francisco Rodríguez (ed.), Migraciones e interculturalidad. Experiencias europeas y latinoamericanas. Varsovia: CESLA.

Schmidt, Susana (2009). De Argentina a España: historias vividas e intercambios imaginados en las migraciones recientes [tesis]. Salamanca, España: Universidad de Salamanca.

Sito, Nilda \& Stuhlman, Luis (1968). La emigración de científicos de la Argentina. San Carlos de Bariloche, Argentina: Fundación Bariloche.

Slemenson, Marta (1970). Emigración de científicos argentinos: organización de un éxodo a América Latina: historia y consecuencias de una crisis político-universitaria. Buenos Aires: Instituto Torcuato Di Tella.

Spivak, Ana \& Hubert, Matthieu (2012). Movilidad científica y reflexividad. De cómo los desplazamientos de los investigadores modelan modos de producir conocimientos. Redes, 18(34), 85-111.

Spivak, Ana \& Hubert, Matthieu. (2014). Volver a casa. Apuntes sobre el programa argentino de cooperación y repatriación de científicos. En Mina Kleiche-Dray \& Daniel Villavicencio (coords.), Cooperación, colaboración científica y movilidad internacional en América Latina. Buenos Aires: CLACSO.

Vives-Gonzalez, Luna (2011): Insiders or outsiders? Argentinean immigrants in $\begin{array}{llll}\text { Spain. Citizenship } & \text { Studies, 227-245. }\end{array}$ https://doi.org/10.1080/13621025.2011.549717

Vessuri, Hebe (1998). La movilidad científica desde la perspectiva de América Latina. En Jorge Charum \& Jean-Baptiste Meyer (eds.), El nuevo nomadismo científico. La perspectiva latinoamericana. Bogotá: ESAP.

Vessuri, Hebe (2007). "O inventamos o erramos". La ciencia como idea-fuerza en América Latina. Buenos Aires: Editorial UNQ.

Webster, Jane \& Watson, Richard (2002). Analyzing the past to prepare for the future: the literature review. MIS Quarterly, 26(2), xxiii-xxiii.

Wimmer, Andreas \& Glick-Schiller, Nina (2002). Methodological nationalism and beyond nation-state building, migrations and the social sciences. Global Networks, 2(4), 301-334. https://doi.org/10.1111/1471-0374.00043

Recibido: 24/06/2020 
Migraciones y movilidades de argentinos y argentinas: revisión crítica de un campo de estudios en desarrollo (1960-2020)

Evaluado: $30 / 08 / 2020$

Versión Final: 07/10/2020 